\title{
Makroökonomische Dynamik von Arbeitsmärkten. Ein Vergleich interner und externer Flexibilitäten in den USA und in Deutschland
}

Die internationale Finanz- und Immobilienkrise hat in den USA ihren Ausgang genommen und eine weltweite Wirtschaftskrise mit tiefen Einbrüchen der gesamtwirtschaftlichen Produktion in den Industrieländern herbeigeführt. Die deutsche Volkswirtschaft war aufgrund ihrer ausgeprägten Abhängigkeit von den Exportmöglichkeiten besonders stark betroffen. Trotz eines tiefen Einbruchs der gesamtwirtschaftlichen Produktion erwies sich der Arbeitsmarkt in Deutschland als erstaunlich robust. In der relativ milden Rezession in den USA gingen dagegen viele Arbeitsplätze verloren, und die Arbeitslosigkeit stieg kräftig an. In diesem Beitrag werden aus einer makroökonomischen Perspektive Gründe für diese national sehr unterschiedlichen Krisenreaktionen am Arbeitsmarkt analysiert.

\section{Einführung}

Ziel dieser Arbeit ist es, eine Vorstellung von den Zusammenhängen und der Dynamik makroökonomischer Größen zu erhalten, die für Arbeitsmärkte in den USA und Deutschland von Bedeutung sind. Wir gehen dabei von der weltwirtschaftlichen Entwicklung in der aktuellen Finanz- und Wirtschaftskrise aus (Abschnitt 2). Dann nehmen wir die Volkswirtschaften der USA (Abschnitt 3) und Deutschlands (Abschnitt 4) unter die Lupe und beschreiben das Zusammenspiel makroökonomischer Variablen in der Krise. Es folgt eine vergleichende Analyse von Volatilität, Dynamik und Persistenz der Variablen in einer längeren zeitlichen Perspektive (Abschnitt 5), wobei auf das Phänomen des „labour-hoarding“ (Abschnitt 6) näher eingegangen und die Stabilität der Ergebnisse untersucht wird (Abschnitt 7). Schließlich wird dargelegt, welche Faktoren die Arbeitszeitflexibilität in Deutschland bestimmen, und was sie in der Krise am Arbeitsmarkt bewirkt haben könnten (Abschnitt 8). Abschnitt 9 zieht ein knappes Fazit.

\section{Die Weltwirtschaft in der Krise}

Die weltweite Wirtschaftskrise erreichte im Jahr 2009 ihren Tiefpunkt. Sie hatte im Jahr 2007 in den USA mit dem Ende einer durch Finanzprodukte erzeugten
Immobilienblase begonnen und sich dann unerwartet schnell und kräftig in der Weltwirtschaft ausgebreitet. So war der Konjunkturzusammenhang zwischen den Industrieländern im Verlauf der 1990er Jahre stärker geworden, und aufstrebende Schwellenländer, deren Konjunkturen in früheren Jahren nicht zeitgleich mit jenen der Industrieländer verliefen, wurden auch von der Krise erfasst. Der internationale Konjunkturzusammenhang dürfte im Ganzen von der Finanzkrise verstärkt worden sein (Dreger et al. 2009). Im Ergebnis brach die Weltwirtschaft im Jahr 2009 in einem lange Zeit nicht beobachteten Maße ein (Dreger et al. 2010). So verringerte sich der Welthandel um rd. $20 \%$ und die weltwirtschaftliche Produktion nahm um $2 \%$ ab. Dabei zeigten sich ausgeprägte Unterschiede zwischen den Ländern.

\section{USA - milde Rezession mit großen Beschäftigungs- verlusten und hoher Arbeitslosigkeit}

In den USA ging die Wirtschaftsleistung schon ab dem 2. Quartal 2008 kräftig zurück. Die konjunkturelle Entwicklung stabilisierte sich im ersten Halbjahr 2009 und zog in der zweiten Jahreshälfte wieder kräftig an (Abbildung 1). Obwohl die weltweite Krise von den Finanz- und Immobilienmärkten in den USA ausgegangen war, verzeichnete die US-Wirtschaft geringere Verluste als die Wirtschaft in den meisten europäischen Ländern. So nahm das Bruttoinlandsprodukt (BIP) in den USA nur um 2,5\% ab. Die Zahl der Jobs schrumpfte jedoch im Verlauf der Jahre 2008 und 2009 stetig und kräftig. Im Durchschnitt des Jahres 2009 ging die Zahl der Beschäftigten (im „non-farmSektor") um 3,7\% gegenüber dem Vorjahr zurück. Auch die durchschnittliche Arbeitszeit wurde im Verlauf der Krise etwas kürzer. Aufgrund des Rückgangs von Beschäftigung und Arbeitszeit nahm das Arbeitsvolumen kräftig ab, und zwar stärker als die gesamtwirtschaftliche Produktion, sodass die Arbeitsproduktivität in den Jahren 2008 und 2009 anstieg. Insbesondere mit der kräftigen Konjunkturerholung im zweiten Halbjahr 2009 gingen hohe Produktivitätssteigerungen einher. Die Arbeitslosigkeit hingegen nahm in den Jahren 2008 und 2009 kräftig zu. Erst im zweiten Halbjahr flachte der Anstieg etwas ab. Der Arbeitsmarkt in den USA reagierte in der Rezession somit frühzeitig und

Philipp Schaz arbeitete 2009 als Gast-
forscher am Institut für Arbeitsmarkt-
und Berufsforschung (IAB), Nürnberg.
Arbeitsschwerpunkte: Industrieökonomik,
Arbeitszeitstruktur im internationalen
Vergleich.
e-mail: philipp.schaz@uni-konstanz.de
Eugen Spitznagel, Dr., ist Leiter der
Forschungsgruppe „Arbeitszeit und
Arbeitsmarkt“ am IAB. Arbeitsschwerpunkte:
Empirische Makroökonomie, Analyse der
Arbeitszeit im gesamtwirtschaftlichen
Zusammenhang, Evaluation arbeitsmarkt-
politischer Maßnahmen.
e-mail: eugen.spitznagel@iab.de


Abb. 1: Entwicklung von makroökonomischen Variablen in den USA 2005-2009*

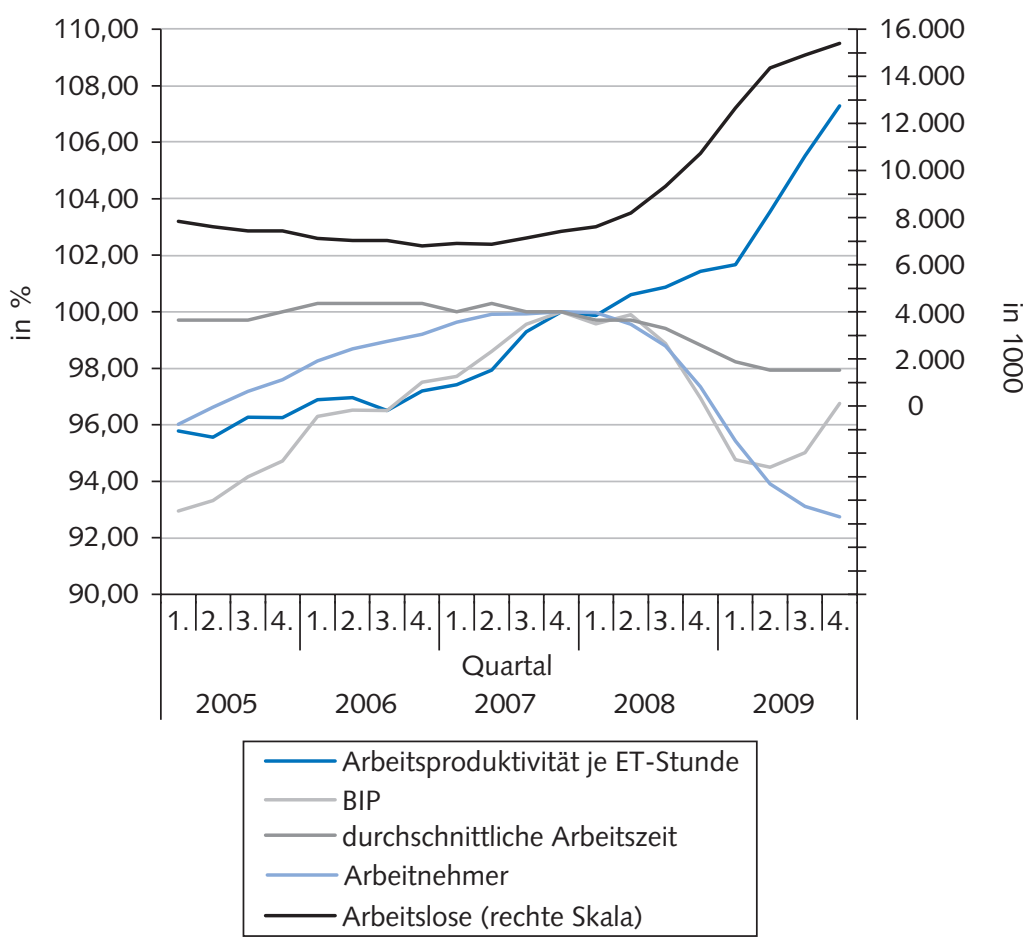

*Indizes bzw. Absolutzahlen; Quartalsdurchschnitte; saisonbereinigt.

Quelle: Bureau of Labour Statistics (BLS); Berechnungen der Autoren.

WSI MITTEILUNGEN

\section{Abb. 2: Entwicklung von makroökonomischen Variablen in Deutschland 2005-2009*}

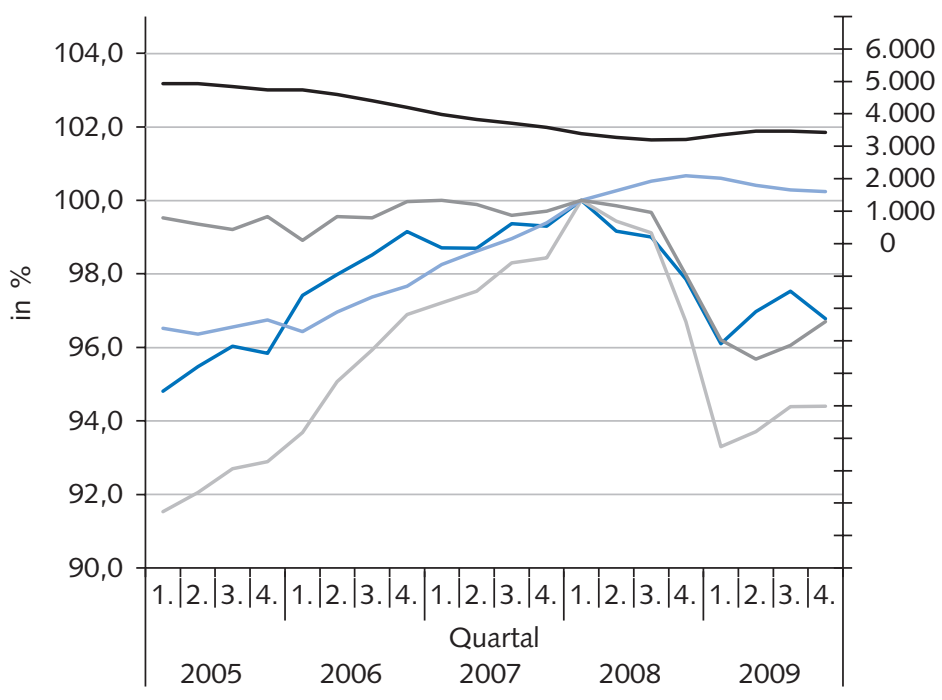

$$
\begin{aligned}
& \text { Arbeitsproduktivität je ET-Stunde } \\
& \text { BIP } \\
& \text { - durchschnittliche Arbeitszeit } \\
& \text { - Arbeitnehmer } \\
& \text { Arbeitslose (rechte Skala) }
\end{aligned}
$$

*Indizes bzw. Absolutzahlen; Quartalsdurchschnitte; saisonbereinigt. massiv. Es ist der Geld- und Fiskalpolitik zwar gelungen, eine schnelle konjunkturelle Erholung einzuleiten (OECD 2010, S. 167-200). Auf dem Arbeitsmarkt war jedoch bis zum Ende des Jahres 2009 keine Wende zum Positiven zu erkennen.

Die heftige Reaktion des Arbeitsmarktes in den USA folgte im Wesentlichen aus dem Prinzip des „hiring and firing“, das in der dortigen Personalpolitik weit verbreitet ist. Aus institutionellen und kulturellen Gründen wird die Beschäftigung in den USA schneller und mit geringeren Kosten der Marktentwicklung angepasst als etwa in Deutschland.

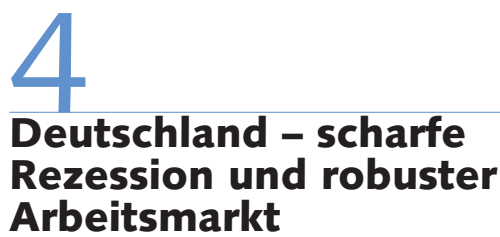

Trotz des Einbruchs der Wirtschaftsleistung um fast 5\% im Jahr 2009 hielt sich der Arbeitsmarkt im Krisenjahr außerordentlich gut. Die Erwerbstätigkeit nahm im Jahresdurchschnitt nur um 17.000 Personen ab, und die Arbeitslosenzahl stieg moderat auf 3,423 Mio. Personen an. Das waren 155.000 oder $4,7 \%$ mehr als im Jahr 2008. Dieser Anstieg konzentrierte sich auf das erste Halbjahr, danach stagnierten die saisonbereinigten Arbeitslosenzahlen von Monat zu Monat oder sanken sogar (Abbildung 2). Allerdings ist Letzteres zum Teil auf Umstellungen in der Statistik zurückzuführen. ${ }^{1}$

Für die robuste Reaktion des Arbeitsmarktes kann eine Reihe von Gründen angeführt werden (Möller 2009):

(1) So befanden sich Wirtschaft und Arbeitsmarkt im Aufschwung, als die Krise Deutschland traf. Insbesondere die Exportwirtschaft hatte vom vorangegangenen Aufschwung kräftig profitiert, und die Unternehmen konnten finanzielle Reserven aufbauen.

1 Zum 1. Januar 2009 wurden die "Maßnahmen zur Aktivierung und beruflichen Eingliederung “ nach § 46 SGB III neu geregelt. Dadurch werden Elemente verschiedener früherer Instrumente zusammengefasst und ähnliche Förderkonzepte in flexibler Kombination ermöglicht. Während Teilnehmer an Eignungsfeststellungs- und Trainingsmaßnahmen bereits zuvor nicht als arbeitslos geführt wurden, gilt dies für Personen, deren Betreuung Dritten übertragen wird, erst seit 2009. Dadurch sind Vergleiche der Arbeitslosenzahl im Zeitverlauf verzerrt (Einzelheiten dazu vgl. Monatsberichte der Bundesagentur für Arbeit (BA) 2009/2010). 
(2) Die Krise entstand nicht in Deutschland, sondern war aufgrund der engen Handelsverflechtungen und Finanzbeziehungen nach Deutschland übertragen worden und betraf primär die Exportwirtschaft. Die vom vergangenen Aufschwung begünstigten Betriebe waren in der Lage und willens, große Teile ihrer Belegschaften während der Krise zu halten.

(3) Die dienstleistungsorientierte Binnenwirtschaft war von der Krise wenig betroffen, und in Bereichen wie Bildung, Gesundheitswesen und Gastronomie wurde weiterhin Beschäftigung aufgebaut (Statistisches Bundesamt 2010).

(4) Die in der Vergangenheit durchgeführten Arbeitsmarktreformen scheinen die Dynamik am Arbeitsmarkt gefördert zu haben (Fahr/Sunde 2009), und die institutionellen Rahmenbedingungen waren eher günstig. So konnten Öffnungsklauseln, die in Tarifverträgen vereinbart worden waren, genutzt werden, um Arbeitszeiten und Löhne der Auftragssituation in den Unternehmen anzupassen. Es wurden weniger Überstunden geleistet und Guthaben auf Arbeitszeitkonten abgebaut. Schließlich trugen die Rücknahme früherer Restriktionen und zusätzliche Anreize beim Kurzarbeitergeld aus konjunkturellen Gründen (\$170 SGB III) zur Stabilisierung der Beschäftigung bei.

Das gesamtwirtschaftliche Arbeitsvolumen aller Erwerbstätigen schrumpfte im Jahr 2009 um 2,6\%. Da die gesamtwirtschaftliche Produktion um 4,7\% geringer ausfiel als im Vorjahr, verringerte sich die Produktivität je Arbeitsstunde um 2,2\% (Statistisches Bundesamt 2010).

\section{Volatilität, Dynamik und Persistenz makroökonomi- scher Größen in den USA und in Deutschland}

Im Folgenden wird untersucht, ob es sich bei den makroökonomischen Entwicklungen 2008/2009 um Besonderheiten der Krisenjahre handelt oder ob vergleichbare Entwicklungen schon früher zu beobachten waren.

\section{Abb. 3: Trendfluktuation des Arbeitsvolumens mit dem \\ Konjunkturzyklus - Deutschland und USA}
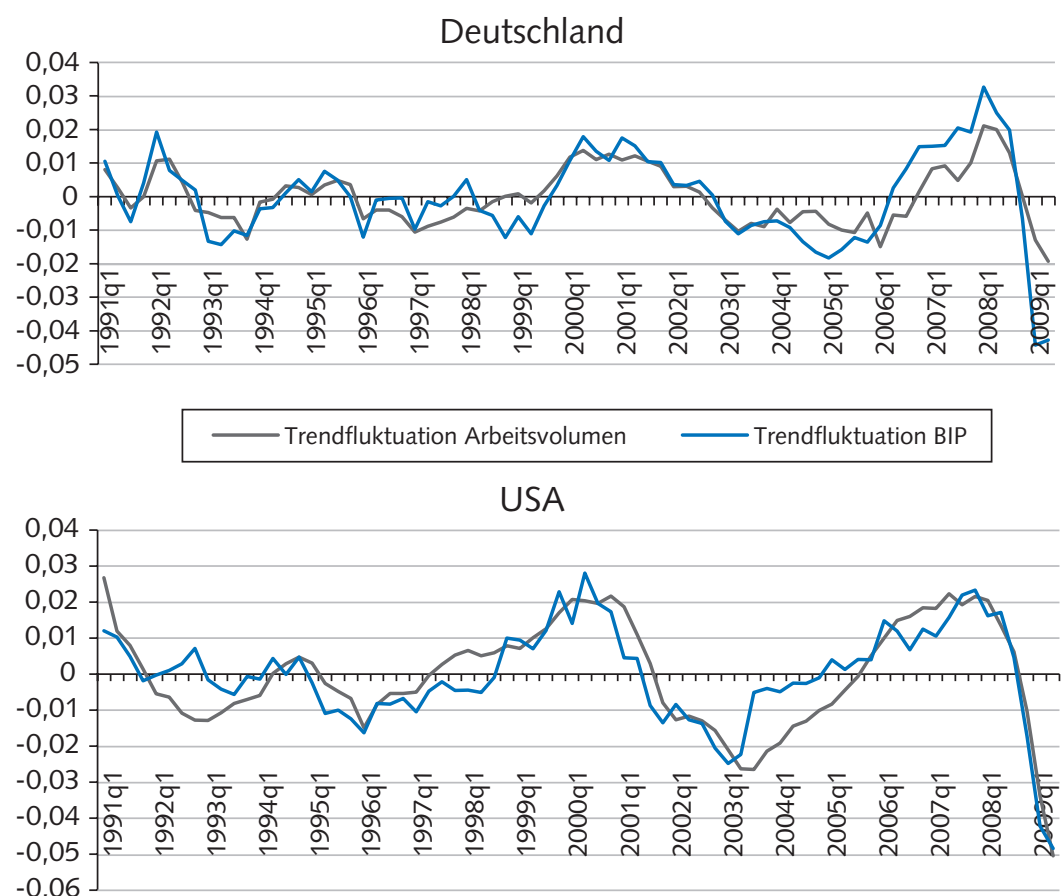

Quelle: Statistisches Bundesamt; BLS; Berechnungen der Autoren.

WSI MITTELUNGEN

\subsection{ZUR METHODE}

Es werden folgende makroökonomischen Größen in die Analyse einbezogen: reales Bruttoinlandsprodukt, Erwerbstätige, Arbeitszeit je Erwerbstätigen, Arbeitsvolumen und Arbeitsproduktivität. Die saisonbereinigten Quartalsdaten für diese Variablen liegen für Deutschland und die USA aus deren nationalen Volkswirtschaftlichen Gesamtrechnungen vor. Die empirischen Ausprägungen der Variablen im Untersuchungszeitraum werden durch die Berechnung von Trends geglättet, um Schwankungen auszugleichen und um das Ausmaß sowie die Unterschiede in der Dynamik messen zu können. Die Zeitreihen der Quartalswerte reichen vom Jahr 1991q1 bis 2009-q2.

Methodisch orientiert sich die Untersuchung an der Vorgehensweise von Shimer (2005). Auf die Zeitreihen wird ein HP-Filter mit dem in der Literatur für Quartalsdaten üblichen Glättungsparameter $\lambda=1600$ angewendet, um aus den Zeitreihen kurzfristige Schwankungen zu eliminieren. Der HP-Filter separiert den Trend einer Zeitreihe von der zyklischen Komponente sowie von irregulären Schwankungen. Die logarithmierte Abweichung der Quartalswerte von ihrem so errechneten Trend misst die
Fluktuation der jeweiligen Variablen. Der natürliche Logarithmus ermöglicht dabei die Darstellung in prozentualen Werten der Größen, als Abweichungen von ihrem so errechneten Trend.

\subsection{ARBEITSVOLUMEN IM KONJUNKTURZYKLUS}

In der makro-ökonomischen Literatur wird der Faktor Arbeit L (für labour) in der Regel durch das Arbeitsvolumen beschrieben. Es misst die Summe aller (bezahlten) Arbeitsstunden, die in einer Volkswirtschaft in einer Periode von allen Erwerbstätigen geleistet werden. Das Arbeitsvolumen (A) errechnet sich als Produkt aus Arbeitszeit je Erwerbstätigen (Z) und Zahl der Erwerbstätigen (E):

$\begin{aligned} & \text { Arbeits- } \\ & \text { volumen } \\ & \text { oder }\end{aligned}=$ Arbeitszeit $\cdot$ Erwerbstätige,
$\quad A=Z \cdot E$

Die Fluktuation des Arbeitsvolumens Arbeit wird gemessen durch die Abweichung ã des tatsächlichen Arbeitsvolumens A vom Trend A* im Untersuchungszeitraum, gemäß

$\tilde{\mathrm{a}}=\ln \left(\frac{\mathrm{A}}{A^{*}}\right)$. 
Tabelle 1: Trendfluktuation des Arbeitsmarktes 1991-2009 (2. Quartal) - Deutschland und USA

\begin{tabular}{|c|c|c|c|c|c|c|c|c|c|}
\hline & & \multicolumn{4}{|c|}{ Deutschland } & \multicolumn{4}{|c|}{ USA } \\
\hline & & $\begin{array}{l}\text { Trend- } \\
\text { fluktuation } \\
\text { BIP }(\tilde{y})\end{array}$ & $\begin{array}{l}\text { Trend- } \\
\text { fluktuation } \\
\text { Arbeits- } \\
\text { volumen (ã) }\end{array}$ & $\begin{array}{c}\text { Trend- } \\
\text { fluktuation } \\
\text { Erwerbstätige } \\
\text { (ẽ) }\end{array}$ & $\begin{array}{c}\text { Trend- } \\
\text { fluktuation } \\
\text { Arbeitszeit } \\
\text { (z̃) }\end{array}$ & $\begin{array}{l}\text { Trend- } \\
\text { fluktuation } \\
\operatorname{BIP}(\tilde{y})\end{array}$ & $\begin{array}{c}\text { Trend- } \\
\text { fluktuation } \\
\text { Arbeits- } \\
\text { volumen (ã) }\end{array}$ & $\begin{array}{c}\text { Trend- } \\
\text { fluktuation } \\
\text { Erwerbstätige } \\
\text { (ẽ) }\end{array}$ & $\begin{array}{c}\text { Trend- } \\
\text { fluktuation } \\
\text { Arbeitszeit } \\
\text { (z̃) }\end{array}$ \\
\hline \multicolumn{2}{|l|}{ Standardabweichung } & 0,0133 & 0,0084 & 0,0065 & 0,005 & 0,0135 & 0,0149 & 0,0121 & 0,004 \\
\hline \multicolumn{2}{|l|}{$\begin{array}{l}\text { Standardabweichung } \\
\text { (relativ zum BIP) }\end{array}$} & 1 & 0,63 & 0,49 & 0,38 & 1 & 1,10 & 0,90 & 0,28 \\
\hline \multicolumn{2}{|l|}{$\begin{array}{l}\text { Vierteljährige } \\
\text { Autokorrelation }\end{array}$} & 0,7353 & 0,7765 & 0,8919 & 0,6080 & 0,7721 & 0,8248 & 0,8319 & 0,7753 \\
\hline \multirow[t]{4}{*}{ Korrelationsmartrix } & $\tilde{y}$ & 1 & 0,8501 & 0,5444 & 0,7183 & 1 & 0,8563 & 0,8160 & 0,7443 \\
\hline & $\tilde{a}$ & - & 1 & 0,8048 & 0,6289 & - & 1 & 0,9809 & 0,7794 \\
\hline & $\tilde{\mathrm{e}}$ & - & - & 1 & 0,0448 & - & - & 1 & 0,6426 \\
\hline & $\tilde{z}$ & - & - & - & 1 & - & - & - & 1 \\
\hline \multicolumn{10}{|c|}{$\begin{array}{l}\text { Anmerkung: Es wurden quartalisierte saisonbereinigte Daten herangezogen. Die Variablen der Trendfluktuation sind als logarithmierte Abweichungen } \\
\text { von ihrem HP-Trend mit Glättungsparameter } \lambda=1600 \text { erfasst. }\end{array}$} \\
\hline \multicolumn{10}{|c|}{ Quelle: IAB; Bureau of Labour Statistics (BLS); Berechnungen des Autoren. } \\
\hline
\end{tabular}

Abbildung 3 zeigt die so gemessene Trendfluktuation des Arbeitsvolumens und des Outputs in Deutschland und den USA. Sie lässt die ausgeprägte prozyklische Entwicklung des Arbeitsvolumens in beiden Ländern erkennen.

\section{STRUKTURELLE ZUSAMMENHÄNGE}

Die Schwankungen des Arbeitsvolumens im Konjunkturverlauf sind ein Charakteristikum von Arbeitsmärkten. So können Friktionen im Search-and-MatchingProzess verhindern, dass Arbeitsuchende und offene Stellen zusammenkommen. Die Ursache solcher Friktionen sind Transaktionskosten verschiedener Art, die zu Rigiditäten führen können. Sie können aus Mindestlohnbestimmungen, Kündigungsschutz, qualifikatorischen und regionalen Diskrepanzen zwischen Angebot und Nachfrage sowie aus Fehlanreizen des Arbeitslosenversicherungssystems entstehen, um nur einige der Ursachen von Transaktionskosten zu nennen. Diese Friktionen beeinflussen die Reagibilität des Arbeitsvolumens hinsichtlich konjunktureller Schwankungen. Die nationalen Ausprägungen dieser Ursachen können zu internationalen Unterschieden in der Reagibilität des Arbeitsvolumens auf konjunkturelle Schwankungen führen.

Aus der Komposition des gesamtwirtschaftlichen Arbeitsvolumens ergeben sich zwei Formen von Reagibilität. Aus seinen Komponenten „Arbeitszeit“ und „Erwerbstätige" erhält man aggregierte Formen von interner und externer Flexibilität des Produktionsfaktors Arbeit.

Hinter der internen Flexibilität steht idealtypisch eine Personalpolitik der Un- ternehmen, welche die Zahl der Erwerbstätigen bei konjunkturellen Schocks unverändert lässt und die Anpassung bei veränderter Auftragssituation über die Arbeitszeit oder/und die Produktivität vollzieht.

Hinter der externen Flexibilität steht dagegen eine Personalpolitik, die Anpassungen über die Zahl der Beschäftigten vornimmt, wenn sich wirtschaftliche Rahmenbedingungen ändern. In der Realität dürften beide Flexibilitätsformen zugleich vorkommen.

Eine nach den Komponenten Arbeitszeit, Erwerbstätige und Produktivität je Stunde differenzierte Analyse verschafft so einen Eindruck, von welchen Formen der Flexibilität die Arbeitsmärkte in den beiden Volkswirtschaften geprägt sind.

Die Standardabweichungen der Trendfluktuationen für die Größen Brutto-

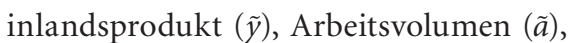

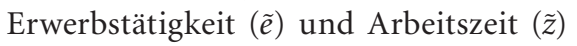
sind für den gesamten Untersuchungszeitraum in Tabelle 1 ausgewiesen.

Die einzelnen Standardabweichungen werden auf jene des Bruttoinlandsprodukts normiert (Tabelle 1, zweite Zeile). Da die so gemessenen Volatilitäten in beiden Volkswirtschaften annähernd gleich sind, bietet sich diese Normierung an. Diese Zahlen beschreiben also die Schwankungen der Arbeitsmarkt-Variablen relativ zur Schwankung des Bruttoinlandsprodukts, was die internationale Vergleichbarkeit erleichtert.

Implizit unterstellt dieser Ansatz, dass die Arbeitsmarktgrößen von der gesamtwirtschaftlichen Produktion abhängen. Das Arbeitsvolumen und das Bruttoinlandsprodukt stehen im Allgemeinen jedoch in einer wechselseitigen
Kausalbeziehung. So hängt der Output vom Arbeitsvolumen ab, wenn man von einer Produktionsfunktion im Sinne einer technischen Beziehung ausgeht. Andererseits folgt aus der Inversion der Produktionsfunktion eine Beschäftigungsfunktion, in der das Arbeitsvolumen vom Output abhängt. Bei Analysen von Produktionspotenzialen und Perioden, in denen Vollbeschäftigung herrschte, ist es zweckmäßig, die Kausalität durch eine Produktionsfunktion zu approximieren. Bei Analysen zyklischer Schwankungen, die mit Unterbeschäftigung einhergehen, ist es zweckmäßig, die umgekehrte Kausalität anzunehmen und zu unterstellen, dass die Arbeitsmarktentwicklung im Wesentlichen von der Dynamik der gesamtwirtschaftlichen Nachfrage und der Produktion getrieben ist. Da in den hier betrachteten Jahren weder in den USA noch in Deutschland Vollbeschäftigung herrschte, sondern die Jahre mit Unterbeschäftigung bei Weitem überwogen, legen wir unserer Analyse einen nachfrageorientierten Ansatz zugrunde und betrachten das Arbeitsvolumen als abgeleitete Größe der gesamtwirtschaftlichen Produktion.

Man sieht, dass die meisten der hier betrachteten makroökonomischen Arbeitsmarktvariablen in den USA volatiler sind als die entsprechenden Größen in Deutschland. So beträgt die Standardabweichung des Arbeitsvolumens in den USA 1,10; in Deutschland hingegen 0,63. Mit einer Standardabweichung von 0,90 sind die Erwerbstätigen in den USA annähernd doppelt so volatil wie in Deutschland, wo der entsprechende Wert 0,49 beträgt. Dagegen ist die Standardabweichung der Arbeitszeit mit 0,28 in den 
USA geringer als in Deutschland $(0,38)$. Das Arbeitsvolumen und die Zahl der Erwerbstätigen sind in den USA somit volatiler, die Arbeitszeit ist weniger volatil als in Deutschland.

Die Persistenz der Trendfluktuationen, also ihr Beharrungsvermögen, lässt sich an den vierteljährlichen Autokorrelationen ihrer Abweichungen vom Trend messen. Diese geben an, wie stark die Trendabweichungen mit ihrem eigenen Vorquartalswert zusammenhängen. Die vierteljährlichen Autokorrelationen zeigen, dass die Fluktuationen von BIP und Arbeitsvolumen in beiden Volkswirtschaften einen in etwa gleich hohen Grad an Persistenz haben. Bei den Komponenten des Arbeitsvolumens zeigen sich jedoch Unterschiede: So ist die Persistenz der Fluktuation der Erwerbstätigenzahl in Deutschland $(0,89)$ geringfügig höher als in den USA $(0,83)$. Das Beharrungsvermögen der Fluktuation der Arbeitszeit hingegen ist in Deutschland $(0,61)$ jedoch deutlich geringer als in den USA $(0,78)$. Die Trendfluktuation der Arbeitszeit in Deutschland verharrt damit seltener auf ihrem Vorquartalsniveau als dies in den USA der Fall ist.

Es ist also zu erkennen, dass die Volatilität des Arbeitsvolumens und seine Persistenz im Konjunkturverlauf in den beiden Volkswirtschaften eine unterschiedliche Struktur haben. In den USA ist die externe Flexibilität stärker ausgeprägt. D.h. Anpassungen erfolgen stärker über die Veränderungen der Erwerbstätigenzahl. Dagegen ist in Deutschland die interne Flexibilität stärker ausgeprägt, d.h. Anpassungen erfolgen stärker über Veränderungen der Arbeitszeit. Die Ergebnisse sind sowohl im Hinblick auf Persistenz wie auch Volatilität über den untersuchten Zeitraum konsistent.

Aktuelle Entwicklungen am Arbeitsmarkt decken sich mit diesen Beobachtungen. So ist das sinkende Arbeitsvolumen im Zuge der Finanz- und Wirtschaftskrise in Deutschland fast vollständig durch Verkürzung der Arbeitszeit erklärt. In den USA hingegen spielte die Arbeitszeit nur eine geringe Rolle, während die Beschäftigung kräftig schrumpfte. Die interne Flexibilität, die in Schwankungen der Auslastung des Arbeitsvolumens bzw. Schwankungen der Produktivität je Stunde zum Ausdruck kommt, wird im Abschnitt 6 analysiert. In Abschnitt 7 überprüfen wir die Stabilität der strukturellen Zusammenhänge in der Zeit seit Ausbruch der Finanzkrise.

\section{DYNAMISCHE ZUSAMMENHÄNGE}

Richtung und Stärke der bisher analysierten Zusammenhänge lassen darauf schließen, dass der Arbeitsmarkt in Deutschland eine höhere interne Flexibilität, der Arbeitsmarkt in den USA hingegen eine höhere externe Flexibilität aufweist.

Dynamische Anpassungsprozesse werden durch Kreuzkorrelationen erfasst. Sie liefern Hinweise darauf, ob das Arbeitsvolumen bzw. seine Komponenten pro- oder kontrazyklisch zum Bruttoinlandsprodukt reagieren und bei wie vielen Quartalen Vorlauf bzw. Nachlauf der lineare Zusammenhang am stärksten ist.

Abbildung 4 zeigt die Reaktion der Trendfluktuation der Arbeitszeit $z^{\sim}$ auf eine positive Trendabweichung des Bruttoinlandsprodukts zum Zeitpunkt 0 und trägt die Korrelationen zwischen $z^{\sim}$ und $y^{\sim}$ zeitverzögert um zehn Quartale vor und nach einem positiven Schock ( $y^{\sim}$ positiv) ab. Die Generierung entsteht über originäre statistische Grunddaten der beiden Volkswirtschaften, wobei das Ausmaß der jeweiligen Outputschocks unterschiedlich ist.
Die Korrelationen 0,74 (USA) und 0,72 (Deutschland) zwischen der Trendfluktuation der Arbeitszeit und des BIP sind in beiden Volkswirtschaften unmittelbar nach dem Schock am stärksten (Tabelle 1). Das Kreuzkorrelationsdiagramm für Deutschland hat jedoch eine schmalere „Taille“ als jenes für die USA. Das bedeutet, dass die Arbeitszeit in Deutschland schneller angepasst wird als in den USA. Die interne Flexibilität durch Änderungen der Arbeitszeit ist somit auch in zeitlicher Hinsicht in Deutschland größer als in den USA.

Ein differenziertes Bild liefern auch die Diagramme der Kreuzkorrelationen der Trendfluktuationen von Erwerbstätigen und BIP. Sie beschreiben die Dynamik der externen Flexibilität bei einem positiven Schock. Abbildung 5 sowie die Werte in Tabelle 1 zeigen, dass die Korrelation in den USA $(0,82)$ weit über jener in Deutschland liegt $(0,54)$. Der stärkere Effekt tritt in den USA sofort ein, wohingegen in Deutschland die stärkste Anpassung mit einer durchschnittlichen Verzögerung von einem Quartal erreicht wird. Gleichzeitig erkennt man, dass sich die Anpassung in

\section{Abb. 4: Dynamische Anpassung der Arbeitszeit bei einem positiven Schock - Deutschland und USA - Kreuzkorrelationsdiagramm}

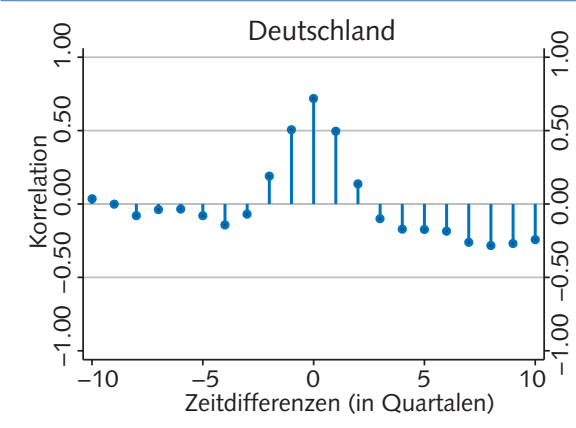

Quelle: IAB; BLS; Berechnungen der Autoren.

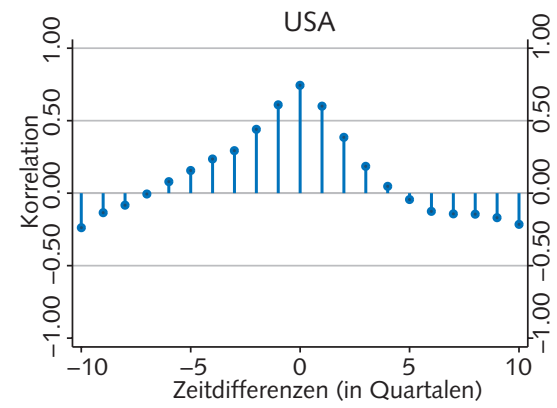

WSI MITTEILUNGEN

\section{Abb. 5: Dynamische Anpassung der Erwerbstätigen bei einem positiven Schock - Deutschland und USA - Kreuzkorrelationsdiagramm}

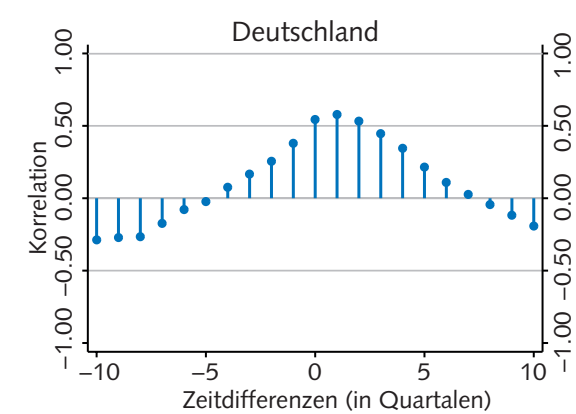

Quelle: IAB; BLS; Berechnungen der Autoren.

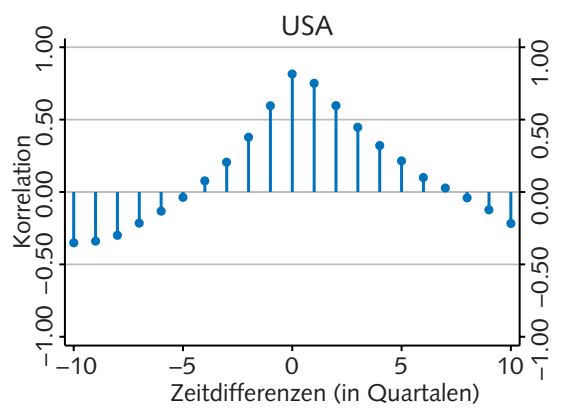

WSI MITTELUNGEN 
Deutschland nur langsam und für die ersten drei Quartale auf einem vergleichbar niedrigen Niveau vollzieht. Der Effekt in den USA hingegen ist unmittelbar nach einem Schock am stärksten und flacht anschließend ab. Die externe Flexibilität ist auf dem Arbeitsmarkt in den USA also nicht nur stärker ausgeprägt als in Deutschland, sondern sie geht auch schneller vonstatten als hierzulande.

\section{Arbeitsproduktivität und "labour-hoarding" im Konjunkturzyklus}

Die wirtschaftswissenschaftliche Literatur enthält eine reiche Diskussion über Veränderungen der Arbeitsproduktivität im Konjunkturverlauf (siehe Baily et al. 2001). Dabei wurde die zunächst a priori plausible Hypothese untersucht, dass in Zeiten eines Abschwungs die Produktivität im Zuge von Entlassungen und Effizienzkalibrierungen steigt, die Arbeitsproduktivität somit antizyklisch sein sollte.

Die Ergebnisse der empirischen Forschung zeigen allerdings ein anderes Bild: Demnach entwickelt sich die beobachtete
Arbeitsproduktivität (je Arbeitsstunde) auf der Aggregatebene prozyklisch. D.h. sie sinkt im Abschwung und steigt im Aufschwung, oder sie steigt in diesen Phasen schwächer bzw. stärker. Dies lässt allerdings nur bedingt eine inhaltliche Interpretation zu, da sich auf makroökonomischer Ebene viele Effekte ausgleichen oder auch kumulieren und verstärken können. Die Richtung und das Ausmaß der Zyklizität von Veränderungen der Arbeitsproduktivität geben jedoch zumindest Hinweise darauf, welche Effekte überwiegen, sodass sich diese Vorgehensweise für eine international vergleichende Analyse eignet.

Als Erklärung für den prozyklischen Effekt führen Baily et al. (2001) neben Externalitäten vor allem das durch Friktionen und institutionelle Faktoren verursachte Horten von Arbeitskräften an. So spielt bei der Entscheidung, einen Arbeitnehmer zu entlassen, zum Beispiel sein firmenspezifisches Humankapital in Form von speziellem Training, seine Vertrautheit mit betriebsorganisatorischen Abläufen eine Rolle, und es sind Spill-over-Effekte auf Kollegen zu bedenken. Im Zuge des demografischen Wandels, der zunehmenden Spezialisierung bei drohender Abwanderung von hochqualifizierten Arbeitskräften und den daraus resultierenden Frik-

\section{Abb. 6: Trendfluktuation der Stundenproduktivität mit dem Konjunkturzyklus - Deutschland und USA}
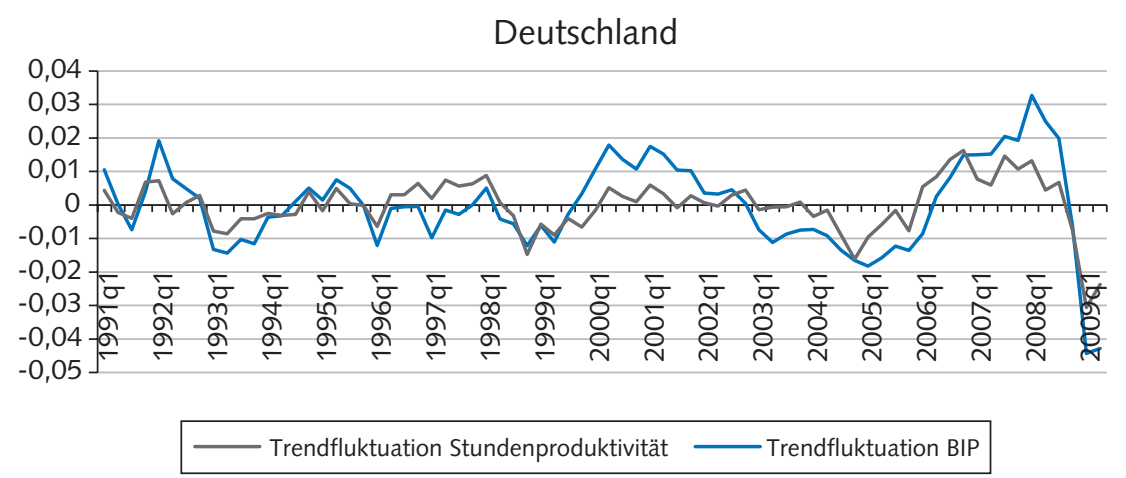

USA

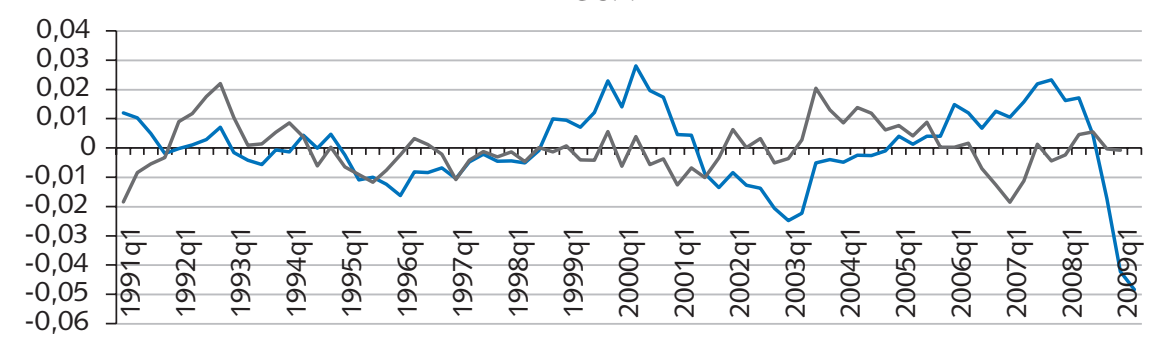

tionen im Search-and-Matching-Prozess könnten die Opportunitätskosten einer Entlassung möglicherweise in den vergangenen Jahren gestiegen sein. Solange der Ertrag des firmenspezifischen $\mathrm{Hu}$ mankapitals höher ist als die Opportunitätskosten einer Kündigung, wird das Unternehmen versuchen, die Beschäftigung auch in wirtschaftlich schlechten Zeiten zu halten. Dies kann durch interne Flexibilität, wie Arbeitszeitverkürzungen, Überstundenabbau, Zwangsurlaub oder Kurzarbeit, erreicht werden. Diese Form der intertemporalen Optimierung hat zur Folge, dass die Produktivität je Beschäftigten prozyklisch verläuft, also wegen Arbeitszeitverkürzungen im Abschwung sinkt und wegen Arbeitszeitverlängerungen im Aufschwung steigt.

Über die Arbeitszeiteffekte hinaus zeigt sich empirisch eine weitere spezifische Form des „Hortens und Ent-Hortens“ von Arbeit. So verändert sich auch die Produktivität je Arbeitsstunde prozyklisch. Dabei sind die Schwankungen hier schwächer ausgeprägt als jene der Arbeitsproduktivität je Beschäftigten, weil die Arbeitszeiteffekte rechnerisch eliminiert sind. Die Prozyklizität der Arbeitsproduktivität je Arbeitsstunde spiegelt die Schwankungen der Auslastung des Arbeitsvolumens im Konjunkturverlauf wider. Um diese Form der internen Flexibilität geht es im Folgenden.

Dem Konzept der Arbeitsproduktivität liegt definitionsgemäß eine einfache Produktionsfunktion der Form $Y=A \cdot P$ zugrunde. Hierbei bezeichnet A das Arbeitsvolumen und P die Arbeitsproduktivität. Beim Berechnen des Trendverlaufs der so definierten Arbeitsproduktivität wird der technische Fortschritt nicht explizit berücksichtigt. Die Fluktuation um den Trend $(\tilde{p})$ interpretieren wir als zyklische Schwankung der Arbeitsproduktivität, die sich aus zeitverzögerten Anpassungsvorgängen ergibt bzw. - bei gegebener Produktion - als Schwankung des Auslastungsgrades des Arbeitsvolumens.

Abbildung 6 zeigt den Verlauf der Trendfluktuation des Bruttoinlandsprodukts $\left(y^{\sim}\right)$ und der Produktivität $\left(p^{\sim}\right)$ für Deutschland und die USA. In Tabelle 2 sind die Entwicklungen während der Jahre 2005 bis 2009 anhand ihrer Ursprungszahlen für Deutschland und die USA dargestellt. Die Bezugsgröße dieser Prozentzahlen ist dabei der HP-Trend. So ergibt sich ein Wert von $100 \%$ wenn die Arbeitsproduktivität (P) gleich ihrem schwankungsbereinigten 
Trend $\left(\mathrm{P}^{\star}\right)$ ist, der approximativ Situationen mit „normaler“, d. h. mittlerer Auslastung der Produktionsfaktoren, beschreibt. Die Werte in Tabelle 2 können somit auch als Auslastungsgrade ( $\left.A^{\star} / A\right)$ des gesamtwirtschaftlichen Arbeitsvolumens interpretiert werden, denn es gilt $\mathrm{P} / \mathrm{P}^{\star}=(\mathrm{Y} / \mathrm{A})$ : $\left(Y / A^{*}\right)=A^{\star} / A$. Dabei symbolisiert $A^{*}$ das bei realisierter Produktion $Y$ gewünschte Arbeitsvolumen.

In Deutschland ist der Auslastungsgrad im Zuge des wirtschaftlichen Aufschwungs in den Jahren 2005 bis 2007 fast stetig angestiegen und erreichte im 1. Quartal 2008 mit 101,84\% seinen höchsten Stand. Mit Beginn der Rezession nahm er stetig ab, erreichte im 1. Quartal 2009 mit 97,81 \% einen Tiefststand und stieg mit der im 2. Quartal einsetzenden wirtschaftlichen Belebung in der Folgezeit leicht an. Vom rezessionsbedingten Rückgang des Auslastungsgrads zwischen Höchst- und Tiefststand um rd. vier Prozentpunkte ist knapp die Hälfte (1,84 Prozentpunkte) als Normalisierung einer in den Jahren des Aufschwungs aufgebauten Arbeitsverdichtung zu interpretieren. Lediglich die verbleibende gute Hälfte (2,19 Prozentpunkte) betrifft die rezessionsbedingte Unterauslastung durch „labour-hoarding“ im Sinne von zeitverzögerter oder unterlassener Anpassung des Arbeitsvolumens an die Entwicklung der Produktion. Dies ist zu berücksichtigen, wenn die lohnpolitischen Implikationen und Konsequenzen der Entwicklung von Produktion, Arbeitsvolumen und Produktivität in der Krise diskutiert werden.

In den USA ging der Auslastungsgrad des Arbeitsvolumens von seinem letzten Höchststand im 4. Quartal 2007 bis zu seinem Tiefststand im 1. Quartal 2009 nur um gut einen Prozentpunkt zurück. Auch wenn in Rechnung gestellt wird, dass die Produktion in den USA nur halb so tief eingebrochen ist wie in Deutschland, ist der Auslastungsgrad während der Krise dort vergleichsweise wenig zurückgegangen. Dies reflektiert die relativ geringe Bedeutung dieser Komponente der internen Flexibilität des Arbeitsmarktes in den USA.

In Deutschland ist ein enger Zusammenhang zwischen der Entwicklung der Arbeitsproduktivität und den konjunkturellen Schwankungen der Produktion zu beobachten, während in den USA auf den ersten Blick kein einheitliches Muster zu erkennen ist (Abbildung 6). Zwar zeichnet sich annähernd ein Gleichlauf dieser Grö-
Ben etwa bis Mitte des Jahres 1998 ab. Aber in der Folgezeit lösen sich die Entwicklungen von diesem Schema.

Ein Blick auf Tabelle 3 bestätigt den Eindruck der beiden Grafiken. So unterscheiden sich Volatilität und Persistenz der Fluktuation der Arbeitsproduktivität in den USA und in Deutschland nicht nennenswert. Aber es zeigt sich ein beträchtlicher Unterschied beim Zusammenhang mit der Konjunktur. So erweist sich die Fluktuation der Arbeitsproduktivität in Deutschland mit einer Korrelation von $\operatorname{Corr}(\tilde{y} ; \tilde{p})=0,8211$ als stark prozyklisch, wohingegen dieser Zusammenhang in den USA mit $\operatorname{Corr}(\tilde{y} ; \tilde{p})=0,0508$ nicht signifikant ist. Ein Vergleich der Kreuzkorrelationsdiagramme bietet an dieser Stelle keine neuen Erkenntnisse, da die Korrelation in den USA nicht signifikant von null verschieden ist. Es zeigt sich lediglich, dass die Anpassung in Deutschland nach einem Quartal am stärksten ist. Die empirische Evidenz deutet somit darauf hin, dass Schwankungen der Produktivität bzw. der Auslastung des Arbeitsvolumens in den USA zumindest seit Ende der 1990er Jahre kein Element interner Flexibilität sind, während sie in Deutschland anhaltend große Bedeutung haben.

Dieses Ergebnis steht im Kontrast zu den Ergebnissen der Forschung über die Zyklizität der Arbeitsproduktivität aus den 1990er Jahren. Der US-amerikanische Arbeitsmarkt scheint seit Mitte des Jahres 1998 kaum mehr von prozyklischen Veränderungen der Arbeitsproduktivität geprägt $\mathrm{zu}$ sein. Eine ceteris-paribus-Schlussfolgerung aus dieser Beobachtung ist, dass der Effekt des „labour-hoarding“ im Sinne von zeitverzögerter Anpassung des Arbeitsvolumens an konjunkturelle Schwankungen

\begin{tabular}{|c|c|c|}
\hline Quartal & Deutschland & USA \\
\hline $2005-1$ & 98,20 & 101,28 \\
\hline $2005-2$ & 98,71 & 100,56 \\
\hline $2005-3$ & 99,11 & 100,81 \\
\hline $2005-4$ & 98,72 & 100,30 \\
\hline $2006-1$ & 100,15 & 100,46 \\
\hline $2006-2$ & 100,57 & 100,05 \\
\hline $2006-3$ & 100,98 & 99,08 \\
\hline $2006-4$ & 101,46 & 99,30 \\
\hline $2007-1$ & 100,87 & 99,01 \\
\hline $2007-2$ & 100,77 & 99,03 \\
\hline $2007-3$ & 101,38 & 99,87 \\
\hline $2007-4$ & 101,23 & 100,03 \\
\hline $2008-1$ & 101,84 & 99,35 \\
\hline $2008-2$ & 100,99 & 99,52 \\
\hline $2008-3$ & 100,84 & 99,22 \\
\hline $2008-4$ & 99,64 & 99,20 \\
\hline $2009-1$ & 97,81 & 98,86 \\
\hline $2009-2$ & 98,73 & 100,10 \\
\hline $2009-3$ & 99,36 & 101,42 \\
\hline $2009-4$ & 98,59 & 102,53 \\
\hline \multicolumn{3}{|c|}{$\begin{array}{l}\text { Anmerkung: Der prozentuale Auslastungsgrad des } \\
\text { Arbeitsvolumens ist der Quotient aus Stundenproduk- } \\
\text { tivität und dessen HP-Trend mit Clättungsparameter } \\
\lambda=1600 \text { ohne natürlichem Logarithmus. }\end{array}$} \\
\hline \multicolumn{3}{|c|}{$\begin{array}{l}\text { Quelle: IAB; Bureau of } \\
\text { Labour Statistics (BLS); } \\
\text { Berechnungen der Autorer }\end{array}$} \\
\hline
\end{tabular}

in den USA stark nachgelassen hat. Dieses Ergebnis kontrastiert mit einer Interpretation der Ergebnisse einer neueren Untersuchung von längerfristigen Entwicklungen in den USA (Davis et al. 2010). Hier wird für die Jahre 1976 bis 2004 eine tendenziell abnehmende Volatilität von ökonomischen Aktivitäten und von Arbeitsmarktvariablen festgestellt und als Ergebnis kompensierender Flexibilitäten und steigender Anpassungskosten gedeutet, die jedoch nicht spezifiziert werden. Und es wird gefolgert, dass die Beschäftigungseffekte von Nachfrageschocks am Arbeitsmarkt in den

\begin{tabular}{|c|c|c|c|c|}
\hline & \multicolumn{2}{|c|}{ Deutschland } & \multicolumn{2}{|c|}{ USA } \\
\hline & $\begin{array}{l}\text { Trendfluktuation } \\
\quad \operatorname{BIP}(\tilde{y})\end{array}$ & 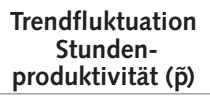 & $\begin{array}{l}\text { Trendfluktuation } \\
\quad \operatorname{BIP}(\tilde{y})\end{array}$ & $\begin{array}{l}\text { Trendfluktuation } \\
\text { Stunden- } \\
\text { produktivität }(\tilde{p})\end{array}$ \\
\hline Standardabweichung & 0,0133 & 0,0079 & 0,0135 & 0,0081 \\
\hline $\begin{array}{l}\text { Standardabweichung } \\
\text { (relativ zum BIP) }\end{array}$ & 1 & 0,59 & 1 & 0,60 \\
\hline $\begin{array}{l}\text { Vierteljährige } \\
\text { Autokorrelation }\end{array}$ & 0,7353 & 0,6290 & 0,7721 & 0,6524 \\
\hline Korrelationsmartrix & 1 & 0,8211 & 1 & $-0,0508$ \\
\hline \multirow[t]{2}{*}{ P-Wert } & & {$[0.000]$} & & [0.6693] \\
\hline & - & 1 & - & 1 \\
\hline \multicolumn{5}{|c|}{$\begin{array}{l}\text { Anmerkung: Es wurden quartalisierte saisonbereinigte Daten heraungezogen. Die Variablen der Trendfluktuation sind als } \\
\text { logarithmierte Abweichungen von ihrem HP-Trend mit Glättungsparameter } \lambda=1600 \text { erfasst; der P-Wert gilt für den T-test } \\
\text { auf signifikanz der Korrelationen. }\end{array}$} \\
\hline \multicolumn{5}{|c|}{ Quelle: IAB; Bureau of Labour Statistics (BLS); Berechnungen der Autoren. } \\
\hline
\end{tabular}


USA deshalb geringer werden. Eine befriedigende Erklärung sowie eine Prüfung der Stabilität dieser Befunde stehen jedoch aus: „A careful assessment of these alternative interpretations is an important topic for future research “(Davis et al. 2010, S. 284).

Der empirische Befund für Deutschland spricht hingegen für eine starke und stabile Prozyklizität der Arbeitsproduktivität und für ein tendenziell hohes Maß an Arbeitskräftehortung. Diese Beobachtungen ergänzen die Befunde aus Abschnitt 5.2. Dort wurde gezeigt, dass der Arbeitsmarkt in Deutschland aufgrund volatiler Arbeitszeiten ein höheres Maß an interner Flexibilität aufweist als der US-Arbeitsmarkt. In Übersee erfolgen Anpassungen an konjunkturelle Wechsellagen weniger über die Arbeitszeit und die Auslastung des Arbeitsvolumens variiert schwächer als hierzulande. Die Anpassungen erfolgen vielmehr über Veränderungen der Erwerbstätigenzahlen. Die Ursachen liegen größtenteils im unterschiedlichen institutionellen Rahmen und ihren ökonomischen Konsequenzen. So sind der Kündigungsschutz sowie die Regulierungen auf den Gütermärkten in Deutschland stärker ausgeprägt als in den USA, und das System der sozialen Sicherung schützt in Deutschland in höherem Maße vor Einkommensverlusten bei Arbeitslosigkeit. Die Relation zwischen den Weiterbeschäftigungskosten bei einer temporären Unterauslastung der Kapazitäten und den Kosten einer Entlassung von Arbeitskräften und ihrer späteren Wiedergewinnung (Bach/Spitznagel 2009; SVR 2009) dürfte deshalb in Deutschland geringer sein als in den USA. Es scheint in den USA einen besonders intensiven Prozess des "hiring and firing“" zu geben, bei dem über den Konjunkturzyklus hinweg Beschäftigungsverhältnisse geschlossen und gelöst werden (vgl. Davis et al. 1997; Davis et al. 2010; OECD 2010 und die dort angegebene Literatur). Die Frage, in welchem Umfang es sich dabei um Arbeitsplätze im betriebswirtschaftlich-technischen Sinne handelt, die im Schumpeter'schen Sinne geschaffen oder zerstört werden, oder um reine Fluktuation, bedarf einer eigenständigen Darstellung und soll hier nicht diskutiert werden.

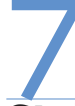 \\ Stabilität der Ergebnisse}

Im Zuge der Finanzkrise unterlag der Arbeitsmarkt starken Veränderungen. Deshalb ist es nötig, die in diesem Beitrag dargelegte Evidenz für einen langen Zeitraum auf ihre Stabilität zu überprüfen.

Abbildung 7 stellt die Standardabweichung der Trendfluktuation der untersuchten Variablen in einem gleitenden Fenster

\section{Abb. 7: Gleitende Fenster der Standardabweichungen Deutschland und USA}
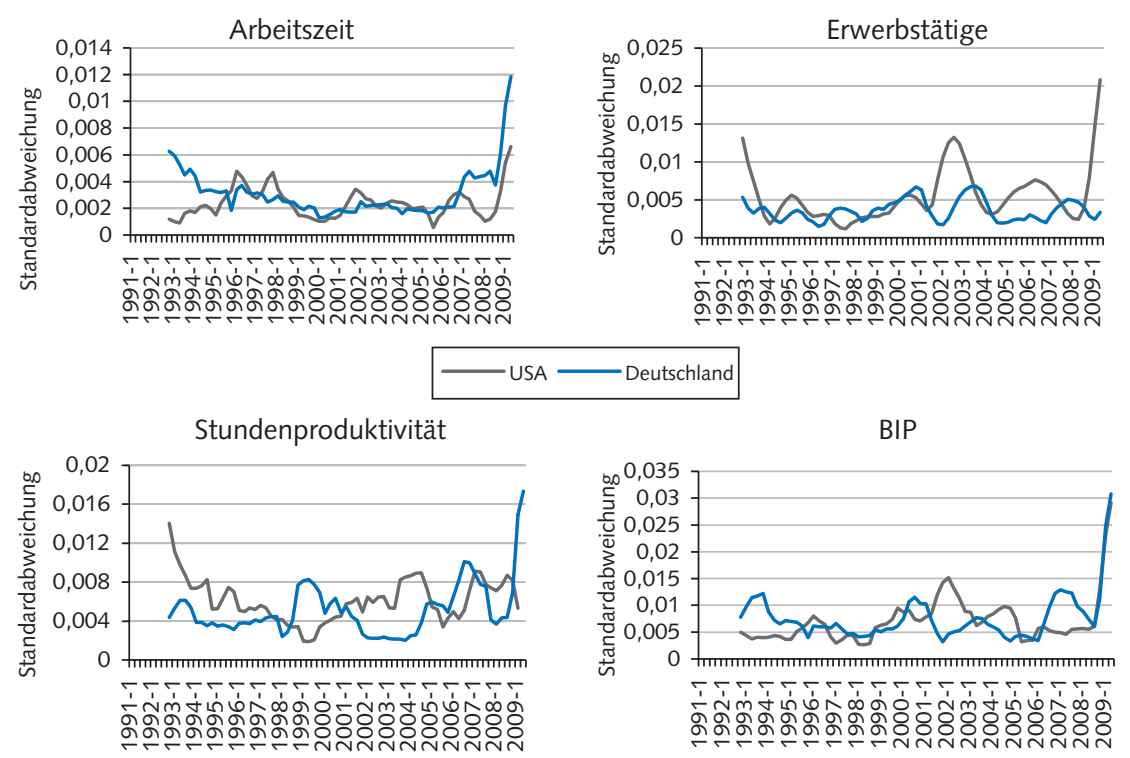

Anmerkung: Das gleitende Fenster trägt zu einem Zeitpunkt die Standardabweichung während der letzten 8 Quartale ab. Erfasst sind die Standardabweichungen der Trendfluktuationen der genannten Variablen.

Quelle: IAB; Bureau of Labour Statistics (BLS); Berechnungen der Autoren.

von acht Quartalen dar. Der erste Punkt bezieht sich auf die Standardabweichung während des Zeitraums 1991/q1-1992/q4. Der Graph schließt sukzessive das nächste Quartal mit ein und lässt gleichzeitig das letzte fallen. Man sieht somit, dass die Standardabweichungen aller Variablen sich im gesamten Zeitraum bis Krisenbeginn in einer gewissen Spannweite bewegen. Dies spricht für die Stabilität der Ausführungen bis zu Beginn der Krise. Zum Rand hin steigt die Volatilität hingegen stark an.

Um die nichtparametrischen Statistiken aus Abschnitt 5 und 6 auf Stabilität zu untersuchen, teilen wir die Daten in zwei Zeiträume auf (bis zum 2. Quartal 2007 und danach). Der Separationszeitpunkt richtet sich nach dem in der Literatur (siehe BIZ 2009) verbreitet genannten Beginn der Finanzkrise. Tabelle 4 weist die für Trendfluktuationen der Variablen Arbeitszeit, Erwerbstätige, Arbeitsvolumen und Stundenproduktivität ermittelten Werte aus. Es bietet sich auch hier an, die Standardabweichungen der Variablen in beiden Ländern auf die Standardabweichung des Bruttoinlandsprodukts in der jeweiligen Periode zu normieren, da diese in absoluten Zahlen in Deutschland und USA annähernd gleich hoch sind (siehe auch Abbildung 7 - BIP).

Betrachtet man die normierten Standardabweichungen in den beiden Perioden, so sieht man, dass die Standardabweichung der Arbeitszeit auch während der Krise in Deutschland $(0,38)$ höher ist als in den USA $(0,23)$, was für eine weiterhin stärker ausgeprägte interne Flexibilität in Deutschland spricht. Die Standardabweichung der Erwerbstätigen ist während der Krise in den USA $(0,71)$ um ein Vielfaches höher als in Deutschland $(0,11)$. Dies spricht für eine noch stärker ausgeprägte externe Flexibilität während der Krise in den USA. Die Aussagekraft dieses strukturellen Zusammenhangs scheint somit auch während der Krise zu bestehen.

Die Persistenz - gemessen als vierteljährige Autokorrelation - der Fluktuation der Arbeitszeit hingegen ist in der Krise in beiden Ländern gleich hoch $(0,67)$. Das Beharrungsvermögen der Fluktuation der Erwerbstätigen ist seit Beginn der Finanzkrise in den USA $(0,60)$ sogar stärker als in Deutschland $(0,39)$. Dies steht im Kontrast zu den Resultaten aus Abschnitt 5.2, wo wir für die Erwerbstätigkeit in Deutschland eine tendenziell höhere und für die Arbeitszeit eine geringere Persistenz als in den USA ermittelt haben. Die geringe 
Zahl an Beobachtungen seit Krisenbeginn beeinflusst allerdings die Robustheit dieser Analyse ebenso wie die Ausnahmesituation während der Krise.

Der dynamische Zusammenhang ist aufgrund der geringen Beobachtungen seit Krisenbeginn nicht mit einer Kreuzkorrelationsanalyse zu überprüfen. Ein Blick auf die Korrelationen mit dem Bruttoinlandsprodukt zeigt jedoch für die Arbeitszeitentwicklung in beiden Ländern etwa das gleiche Bild. Wie aus den Abbildungen 1 und 2 ersichtlich ist, liegt dies daran, dass der stärkere Rückgang der Arbeitszeit in Deutschland mit einem stärkeren Rückgang des Bruttoinlandsprodukts einherging als in den USA. Die Erwerbstätigen hingegen weisen während der Krise in den USA eine stärkere Korrelation auf $(0,98)$ als in Deutschland $(0,74)$, was für eine ausgeprägte externe Flexibilität in den USA spricht und sich mit der Aussage für den gesamten Zeitraum deckt.

Die in Abschnitt 6 gefundene Prozyklizität der Stundenproduktivität ist auch in der Krise in Deutschland $(0,97)$ zu ermitteln. In den USA $(0,03)$ ist sie hingegen nicht festzustellen. Dies spricht somit dafür, dass die interne Flexibilität über die Produktivitätskomponente in Deutschland auch während der Krise stark ausgeprägt war.

Es lässt sich also erkennen, dass die in diesem Beitrag ermittelten Zusammenhänge im Wesentlichen auch während der Finanzkrise zu finden sind. Die Veränderung der Persistenz sowie das Fehlen von Kreuzkorrelationen während des kurzen Zeitraums seit Beginn der Krise lassen derzeit allerdings nur einen bedingten Schluss auf Veränderungen im Ausmaß an interner und externer Flexibilität während der Finanzkrise zu.

\section{Arbeitszeitflexibilität in Deutschland während der Rezession}

Die flexiblen Arbeitszeiten haben die Wucht der Wirtschaftskrise am deutschen Arbeitsmarkt im Jahr 2009 großenteils abgefangen. Im Folgenden wird dargelegt, welche Komponenten der Arbeitszeit diese ausgeprägte Flexibilität erzeugt haben.

Die durchschnittliche Jahresarbeitszeit der Arbeitnehmer verringerte sich im Jahr 2009 um 41,3 Stunden oder 3,1 \% (Spitz-

\begin{tabular}{|c|c|c|c|c|c|c|}
\hline & \multicolumn{3}{|c|}{ Deutschland } & \multicolumn{3}{|c|}{ USA } \\
\hline & $\begin{array}{l}\text { 1991/Q1- } \\
2007 / Q 2\end{array}$ & $\begin{array}{l}2007 / Q 3- \\
2009 / Q 2\end{array}$ & Gesamt & $\begin{array}{l}\text { 1991/Q1- } \\
\text { 2007/Q2 }\end{array}$ & $\begin{array}{l}2007 / Q 3- \\
2009 / Q 2\end{array}$ & Gesamt \\
\hline \multicolumn{7}{|l|}{ Standardabweichung } \\
\hline BIP & 0,010 & 0,031 & 0,013 & 0,011 & 0,029 & 0,014 \\
\hline $\begin{array}{l}\text { Arbeitszeit } \\
\text { (rel. zum BIP) }\end{array}$ & 0,36 & 0,38 & 0,38 & 0,32 & 0,23 & 0,28 \\
\hline $\begin{array}{l}\text { Erwerbstätige } \\
\text { (rel. zum BIP) }\end{array}$ & 0,63 & 0,11 & 0,49 & 1,02 & 0,71 & 0,90 \\
\hline $\begin{array}{l}\text { Arbeitsvolumen } \\
\text { (rel. zum BIP) }\end{array}$ & 0,74 & 0,48 & 0,63 & 1,22 & 0,94 & 1,10 \\
\hline $\begin{array}{l}\text { Stundenproduktivität } \\
\text { (rel. zum BIP) }\end{array}$ & 0,62 & 0,56 & 0,59 & 0,80 & 0,12 & 0,60 \\
\hline \multicolumn{7}{|l|}{ Autokorrelation } \\
\hline Arbeitszeit & 0,50 & 0,67 & 0,61 & 0,79 & 0,67 & 0,78 \\
\hline Erwerbstätige & 0,89 & 0,39 & 0,89 & 0,90 & 0,60 & 0,83 \\
\hline \multicolumn{7}{|l|}{ Korrelation } \\
\hline Corr (BIP; Arbeitszeit) & 0,43 & 0,97 & 0,72 & 0,62 & 0,99 & 0,74 \\
\hline Corr (BIP; Erwerbstätige) & 0,66 & 0,74 & 0,54 & 0,74 & 0,98 & 0,82 \\
\hline $\begin{array}{l}\text { Corr (BIP; Stunden- } \\
\text { produktivität) }\end{array}$ & 0,70 & 0,97 & 0,82 & $-0,07$ & 0,03 & $-0,05$ \\
\hline \multicolumn{7}{|c|}{$\begin{array}{l}\text { Anmerkung: Es wurden quartalisierte saisonbereinigte Daten herangezogen. Die Variablen sind als logarithmierte } \\
\text { Anweichungen von ihrem HP-Trend mit Clättungsparameter } \lambda=1600-\text { also als } \\
\text { Trendfluktuation - erfasst. }\end{array}$} \\
\hline Quelle: IAB; Bureau of Labour & $\mathrm{CC}(\mathrm{DLS}) ; \mathrm{B}$ & antserts & en. & & जvin & LUNGEN \\
\hline
\end{tabular}

nagel/Wanger 2009a). So stieg die Zahl der Kurzarbeiter 2009 sprunghaft an und lag im Jahresdurchschnitt bei 1,143 Mio. Gut ein Drittel ihrer normalen Arbeitszeit fiel aus. Auf die Gesamtheit der Arbeitnehmer umgerechnet sind im Durchschnitt für jeden Arbeitnehmer im Jahr 2009 15,2 Arbeitsstunden durch Kurzarbeit ausgefallen, 13,4 Stunden mehr als im Jahr 2008. Die Guthaben auf den Arbeitszeitkonten sind im Jahresverlauf 2009 um durchschnittlich 8,4 Stunden je Arbeitnehmer abgeschmolzen. Kräftig abgenommen hat auch die bezahlte Mehrarbeit. Je Arbeitnehmer wurden 7,9 bezahlte Überstunden weniger geleistet als im Jahr 2008. Ähnlich große Veränderungen bei diesen flexiblen Komponenten der Arbeitszeit hat es in früheren Jahren nicht gegeben. Auch die normale Wochenarbeitszeit der Vollzeit- und Teilzeitbeschäftigten wurde in der Krise kürzer. So haben krisenbedingte, temporäre Verkürzungen 10,1 Stunden und der Anstieg der Teilzeitarbeit 7,5 Stunden zum Rückgang der durchschnittlichen Jahresarbeitszeit beigetragen. Die Zahl der in Teilzeit beschäftigten Arbeitnehmer (inklusive der geringfügig Beschäftigten) ist im Jahr 2009 um gut 270.000 $(+2,3 \%)$ gestiegen, während fast 270.000 Vollzeitjobs verloren gingen $(-1,1 \%)$. Dies ist vor allem mit der Tatsache zu erklären, dass Teilzeitarbeit in den vom Konjunktureinbruch stark betroffenen Branchen der Industrie vergleichsweise selten ist; in den bisher weniger betroffenen Bereichen privater, sozialer und öffentlicher Dienstleistungen liegt der Teilzeitanteil dagegen deutlich höher (Spitznagel/Wanger 2009b).

Im Ganzen wurden im Jahr 2009 je Arbeitnehmer durchschnittlich 1.309 Arbeitsstunden geleistet. Das sind - wie bereits erwähnt $-3,1 \%$ weniger als im Jahr 2008. Die Arbeitszeit der Selbstständigen und mithelfenden Familienangehörigen veränderte sich hingegen kaum (Spitznagel/Wanger 2009a).

Die Verkürzung der gesamten Arbeitszeit entspricht rechnerisch rund 1,2 Mio. Beschäftigungsverhältnissen, die so gesichert wurden. Anders als in der öffentlichen Diskussion oft vermutet (Zimmermann 2010), war das nicht hauptsächlich auf die Kurzarbeit zurückzuführen, denn sie erklärt nur etwa ein Drittel des Entlastungseffekts. Zwei Drittel des Entlastungseffekts sind anderen Elementen der Arbeitszeitflexibilität zuzuschreiben, wie Arbeitszeitkonten, Überstunden, temporären Verkürzungen der tariflichen Arbeitszeit und der zunehmenden Teilzeitbeschäftigung.

\section{Fazit}

Die Finanz- und Wirtschaftskrise (20072009) hat insbesondere die hoch entwi- 
ckelten Volkswirtschaften getroffen. Das deutsche Konzept der Krisenbewältigung ging bis jetzt auf. Die in hohem Maße flexiblen Arbeitszeiten und der massive Einsatz der Kurzarbeit haben den Arbeitsmarkt in der Krise stabilisiert. Da die meisten Unternehmen die Krise offensichtlich als vorübergehend und nicht als Strukturkrise verstanden haben, hielten sie trotz eines starken Einbruchs der Aufträge und der Produktion ihre gut ausgebildeten und eingearbeiteten Arbeitskräfte. Sowohl die Stärke als auch die Dynamik der Arbeitszeitveränderungen sowie die prozyklischen Schwankungen der Produktivität je Arbeitsstunde sprechen für ein hohes $\mathrm{Maß}$ an interner Flexibilität des Arbeitsmarktes in Deutschland. Diese Flexibilität hängt von tariflichen und arbeitsmarktpolitischen Rahmenbedingungen und von einem gut funktionierenden Dialog zwischen den Sozialpartnern ab. In den
USA ist hingegen die externe Flexibilität stärker ausgeprägt als in Deutschland. Dort tendieren die Unternehmen mehr dazu, Anpassungen über die Zahl ihrer Beschäftigten zu vollziehen, wenn sich die Wirtschaftslage ändert. Darauf lassen sowohl die Schwankungen des Arbeitsvolumens und seiner Komponenten als auch die Azyklizität der Arbeitsproduktivität schließen.

\section{LITERATUR}

Bach, H.-U./Spitznagel, E. (2009): Betriebe zahlen mit - und haben etwas davon, IAB-Kurzbericht 17

Baily, M. N./Bartelsman, E. J./Haltiwanger, J. (2001): Labor Productivity: Structural Change and Cyclical Dynamics, in: The Review of Economics and Statistics 3, S. 420-433

Bank für Internationalen Zahlungsausgleich (BIZ) (2009): Jahresbericht 79, Basel

Bundesagentur für Arbeit (BA) (2009/2010): Monatsberichte, http://www.pub.arbeitsagentur.de/hst/services/statistik/interim/ arbeitsmarktberichte/monatsamb/berichte.shtml.

Cho, J.-O./Cooley, T. F. (1994): Employment and Hours over the Business Cycle, in: Journal of Economic Dynamics and Control 2, S. $411-432$

Davis, S. J./Haltiwanger, J.C./Schuh, S. (1997): Job creation and destruction, in: The MIT Press, Cambridge/Massachusetts/London, S. $125-152$

Davis, S.J./Faberman, R.J./Haltiwanger, J.C./Jarmin, R./Miranda, J. (2010): Business volatility, job destruction, and unemployment, in: American Economic Journal: Macroeconomics 2, S. 259-287 Dreger, Ch./Belke, A./Bernoth, K./Brenke, K./Kooths, S./Kuzin, V./ Weber, S./Zinsmeister, F. (2009): Nach dem Sturm: Schwache und langsame Erholung - Frühjahrsgrundlinien 2009, DIW-Wochenbericht 15-16 Dreger, Ch./Belke, A./Bernoth, K./von Blankenburg, K./Bremus, F./ Brenke, K./Colavecchio, R./Fichtner, F./Hagedorn, H./Kuzin, V. (2010): Frühjahrsgrundlinien 2010, DIW-Wochenbericht 15 Fahr, R./Sunde, U. (2009): Did the Hartz Reforms speed-up the matching process? A macro-evaluation using empirical matching functions, in: German Economic Review 3, S. 284-316

Fuchs, J./Hummel, M./Klinger, S./Spitznagel, E./Wanger, S./Zika, G. (2010): Die Spuren der Krise sind noch länger sichtbar, IAB-Kurzbericht 3 , Nürnberg
Hart, R. A./Malley, J. R. (1999): Procyclical Labour Productivity: A Closer Look at a Stylized Fact, in: Economica 264, S. 533-550 Möller, J. (2009): The German labor market response in the world recession - demystifying a miracle, in: Zeitschrift für Arbeitsmarkt- und Berufsforschung 4, S. 325-336

Organisation für wirtschaftliche Zusammenarbeit und Entwicklung (OECD) (2009): Economic Outlook, Paris

Organisation für wirtschaftliche Zusammenarbeit und Entwicklung (OECD) (2010): Employment Outlook 2010: Moving beyond the job crisis, Paris

Sachverständigenrat zur Begutachtung der gesamtwirtschaftlichen Entwicklung (SVR) (2009): Die Zukunft nicht aufs Spiel setzen, Jahresgutachten 2009/2010, Wiesbaden

Shimer, R. (2005): The Cyclical Behavior of Equilibrium unemployment and Vacancies, in: American Economic Review 1, S. 25-49

Spitznagel, E./Wanger, S. (2009a): Flexible Arbeitszeiten und Kurzarbeit bremsen Jobverluste, Pressemitteilung des IAB vom 4.8. (Daten aktualisiert)

Spitznagel, E./ Wanger, S. (2009b): Vollzeitbeschäftigte sind stärker von der Krise betroffen als Teilzeitbeschäftigte, Pressemitteilung des IAB vom 9.9. (Daten aktualisiert)

Statistisches Bundesamt (2010): Volkswirtschaftliche Gesamtrechnungen, Wiesbaden

Vecchi, M. (2000): Increasing Returns, Labour Utilization and Externalities: Procyclical Productivity in the United States and Japan, in: Economica 1, S. 229-244

Zimmermann, K. (2010): Kurzarbeit: Nützlich in der Krise, aber nun den Ausstieg einleiten, DIW-Wochenbericht 16 